\title{
Oportunidades para la Primera Infancia
}

\section{Opportunities for early childhood}

\author{
Catalina Trujillo Vanegas \\ Magíster en Educación. Universidad Surcolombiana. Neiva. Colombia. \\ catruva73@yahoo.com.co
}

\section{Resumen}

El artículo de investigación da cuenta del análisis de diversos programas que atienden la población en primera infancia en el municipio de Neiva, con el fin de evidenciar las acciones y prácticas que entidades públicas y privadas han implementado para el desarrollo integral de esta población.

Palabras claves: Primera infancia, investigación, atención, educación, desarrollo, programas, entidades públicas y privadas.

\section{Abstract}

The research article considers the analysis of various programmes for children in early childhood in the municipality of Neiva, with the aim of demonstrating the measures and practices that both public and private sector entities have implemented for the integral development of this population group.

Keywords: Early childhood, investigation, attention, education, development, programmes, public and private sector entities

En el reconocimiento investigativo de programas que atendieran la población de primera infancia en el municipio de Neiva, estudiantes de la Licenciatura en Pedagogía Infantil de la Universidad Surcolombiana, realizaron diversos proyectos que dan cuenta de las acciones y prácticas que entidades públicas y privadas han implementado para el desarrollo integral de esta población.

Las investigaciones que contienen un análisis fuertemente enmarcado en la pertinencia de los servicios ofertados, permiten visibilizar la perspectiva y atención puesta en las necesidades y carencias que posterior-mente influyen en su formación, pues en esta etapa de crecimiento los pequeños empiezan a desarrollar habilidades sensoriales, psicomotrices y lingüísticas, las cuales les permitirán poseer todas las bases y facultades físicas y mentales que les han de posibilitar desenvolverse en el mundo.
La atención, cuidado y educación a la primera infancia es concebida entonces como una estrategia efectiva para disminuir la vulnerabilidad propia de los niños y ayudar a nivelar algunas diferencias económicas y sociales que se presentan en el país. "La primera infancia es la etapa del ciclo vital en las que se establecen las bases para el desarrollo cognitivo, emocional y social del ser humano. Comprende la franja poblacional que va de los cero a los seis años de edad $^{\text {"1 }}$.

En este sentido, develando la importancia e impacto que tiene sobre los niños y niñas los primeros años de vida, la incidencia de su entorno, las pautas de crianza que se establecen, los hábitos alimenticios, la protección e interacción humana; se caracterizó a través de los resultados de siete investigaciones ${ }^{2}$, las instituciones públicas y privadas que prestan sus servicios en la ciudad de Neiva en pro de esta población y el impacto sobre su aprendizaje y constitución del ser.

1 - Código de la infancia y adolescencia. Art. 29 Ley 1098 del 2006

2 - 1. Programas de atención a la primera infancia en la ciudad de Neiva; 2 . Una oportunidad para crecer,

"Bibliotecas: espacios no solo para las lecturas"; 3. La primera infancia ino está de primera!; 4. Una nueva perspectiva de la primera infancia;

5. Programas que se implementan en los centros de estimulación temprana y recreación en la ciudad de Neiva hacia el desarrollo integral de la primera infancia; 6. Fundaciones de la ciudad de Neiva en el departamento del Huila que desarrollan programas de atención a la primera infancia; 7. La primera infancia es de vital importancia, por lo tanto conozcamos los programas que se desarrollan en el Instituto Colombiano de Bienestar Familiar de Neiva. 
En primera instancia se abordó un marco general de antecedentes nacionales, en los que se encuentra que desde 1989 se declara en el país la voluntad política de proteger y cuidar a los niños y niñas, especialmente a aquellos que se encuentran en situación vulnerabilidad, a través de la firma de la Convención de los Derechos del Niño.

Históricamente los niños de 0 a 6 años de edad eran atendidos por el sector privado o por el Instituto Colombiano Bienestar Familiar-ICBF pero no formaban parte del sistema educativo formal. A partir de la Ley 115 de 1994 se implementó el Grado Cero para niños de 5 años en las instituciones educativas públicas y dar cumplimiento a la Ley ${ }^{3}$.

En la actualidad, en el Plan Nacional de Desarrollo 2010-2014 "Prosperidad para todos", se instaura la Estrategia de Cero a Siempre que busca contrarrestar los factores asociados al abandono del sistema educativo y la desnutrición, con la creación de programas de atención y cuidado, salud, derecho y deber a la educación, y orientación a los padres de familia para la superación de barreras y condiciones sociales. Una iniciativa que logró poner en sintonía las acciones macro y micro hacia la promoción y garantía del desarrollo de niños y niñas.

En el Huila, el Plan de Atención Integral a la Primera Infancia "Familia PiHuila, ¡Todos Corazón por la Primera Infancia!", tiene como objetivo global "ofrecer asistencia técnica y apoyo en cuanto a acciones coordinadas, intersectoriales, de movilización, caracterización, planeamiento, implementación y evaluación que satisfagan necesidades esenciales para preservar la vida, como otras que reflejan la relación con el desarrollo y aprendizaje humano, acorde a características, necesidades e intereses tanto permanentes como variables en el contexto de la Primera Infancia Huilense".

Ahora bien, estos lineamientos departamentales están dirigidos y se reflejan en entidades de salud y educación que han modificado el contexto. Particularmente, en el caso de la ciudad de Neiva, el municipio "promueve y propicia la garantía de los derechos a la primera infancia y su diversidad, a través del desarrollo de la política pública de atención integral diferenciada, con cobertura, pertinencia, calidad, eficiencia y equidad".

Teniendo conocimiento de dichas acciones oficiales, el propósito de las investigaciones realizadas consistió en evidenciar la pertinencia y funcionalidad de los programas que atienden la primera infancia en Neiva y la idoneidad del personal que forma, desarrolla las actividades e interactúa con los niños y niñas.

La metodología de las investigaciones se planteó de manera cualitativa con el fin de hacer registros narrativos de los fenómenos estudiados mediante técnicas de tipo etnográfico y recolección de información a través de entrevistas y cuestionaros. La población objeto de investigación fueron las siguientes:

- Bibliotecas: Biblioteca Departamental Olegario Rivera, UIS del Norte y UIS del Sur de Comfamiliar Huila; y la Biblioteca Juan Sábalo.

- Cooperativas: Coonfie y Ahorro y crédito del futuro.

- Empresas Privadas: Pequeñín, Comfamiliar, Almacenes Éxito y Cencosud.

- Centros de estimulación temprana y recreación: Castillo mágico, Iwannabe y Baby'art.

- Fundaciones: Fundación Corazón Valiente, Fundación Hermato Oncología del Huila y Fundación Uniendo Sueños.

- ICBF: Hogares infantiles de Santa Inés, Guacirco, y Guambitario.

\section{Resultados}

Bibliotecas: Biblioteca Departamental Olegario Rivera, UIS del Norte, UIS del Sur y Biblioteca Pública Juan Sábalo.

En el estudio investigativo con las bibliotecas se buscó establecer y conocer el contenido de los programas dirigidos a la primera infancia, concibiendo que en esta etapa se estructuran aprendizajes, capacidades y competencias que requieren de las herramientas necesarias para que niños y niñas alcancen diversos conocimientos.

Así desde la época de lactancia, el niño comienza su proceso de aprendizaje, estructuración del pensamiento y formas de expresión que con el tiempo generan una concientización cultural y conductual. En este ámbito familiar donde crece el niño se instauran las primeras y decisivas pautas de comportamiento, identidad y personificación, que posteriormente serán transformadas socialmente en todas sus dimensiones y manifestaciones.

En la actualidad, escenarios como las bibliotecas y actores que la integran, aportan considerablemente a la configuración del saber de niños y niñas: cultura de la lectura y escritura, suministro de información y creación e imaginación.

Entre los 0 y los 6 años se inicia ese momento de la vida que llamamos primera infancia y ocurren los momentos clave en nuestra relación con el lenguaje. Aprendemos a comunicarnos, en la medida en que alguien nos lee y le otorga sentido a nuestros gritos, a nuestros llantos. Aprendemos a hablar después de mucho tiempo de robar voces, de sentir cómo suenan y cómo cantan. Nos ubicamos en el mundo de lo simbólico $y$, finalmente, nos acercamos al código escrito. Yo creo que no hay tres momentos más importantes en nuestra relación con el lenguaje que comunicarnos, entrar al

3 - El marco legal para la primera infancia también se engloba en la Constitución Política, Ley 1098 de 2006, Ley 1295 de 2009. 
lenguaje oral e ingresar a la lengua escrita. Todo esto crea un piso, un nido pleno de significación, entrecruzado de afecto y desvinculado de una actividad que luego se vuelve académica, alfabetizadora. Allí se construyen las bases de la casa imaginaria. (Margolis, 2013)

En la ciudad de Neiva quienes asumen el rol de la casa imaginaria son la Biblioteca Pública Departamental Olegario Rivera que cuenta con la Sala Infantil, un espacio abierto para niños y jóvenes, quienes encuentran en los diversos libros una puerta a la imaginación, y el Aula Tecnológica para Sordos ciegos, que atiende niños y niñas con discapacidad; las Bibliotecas UIS del Norte y UIS del Sur de Comfamiliar del Huila que tienen como propósito incentivar en la población infantil y juvenil el hábito de la lectura y la comprensión de los diferentes géneros literarios, acercando la realidad mágica literaria a su entorno; y la Biblioteca Juan Sábalo, ubicada en la Universidad Surcolombiana.

El análisis y la interpretación de la información recogida en cada uno de estos escenarios permitieron develar la existencia de programas de gran impacto. En las bibliotecas públicas de Comfamiliar del Norte y Sur se encuentran: las "Bebetecas" en donde se brinda estimulación a la primera infancia y "Los bebes si saben leer" un programa que fomenta el hábito lector, adicionalmente estas bibliotecas cuentan con convenios con la Universidad Surcolombiana específicamente con el programa de Pedagogía Infantil, el Banco de la República y la Secretaria de Cultura Municipal y Departamental

De igual forma la biblioteca Departamental Olegario Rivera maneja el programa "Necesidades educativas especiales" atendiendo a niños con cualquier tipo discapacidad. Paralelamente, en la Biblioteca Juan Sábalo de la Universidad Surcolombiana se manejan talleres de lectura, escritura y manualidades a cargo de las practicantes de Licenciatura en Pedagogía Infantil.

En cada una de estas bibliotecas, se realizó la caracterización de las personas encargadas de los programas, para el caso de las bibliotecas públicas del norte y sur de Comfamiliar, las directivas son bibliotecólogas; en la biblioteca departamental la encargada es licenciada en pedagogía infantil y especialista en Educación especial, con formación en el manejo de la población sorda y ciega. Por otro lado en la biblioteca Juan Sábalo la encargada es licenciada en lengua castellana y cuentan con apoyo de estudiantes practicantes bajo la orientación de un asesor cuya especificidad es el trabajo con niños.

\section{Cooperativas: Coonfie ahorro y crédito del futuro}

Otro de los actores que se suman a las iniciativas que potencian las capacidades de los menores de 6 años son las cooperativas. Estas entidades se han encargado de identificar la situación y necesidades presentes en los niños para promover acciones basadas en el juego, el arte y la literatura favoreciendo la participación e inclusión social. Basado en lo anterior, se indagó acerca de los programas de prevención, protección y atención que ofrecen las cooperativas pues

Cuando los niños y las niñas llegan a la escuela es demasiado tarde para muchos de ellos, especialmente para aquellos que presentan problemas de desnutrición, salud, abandono, educación inicial precaria, o han crecido en ambientes maltratadores, de violencia intrafamilar o social. Bajo este argumento se consideraría que la educación de buena calidad para todos es el camino hacia la equidad y que la atención a la primera infancia es importante en tanto determina y garantiza los resultados en las trayectorias escolares futuras. (Castañeda, 2011, p. 2)

En correlación, mediante trabajo de campo se evidenció que en Neiva hay 14 Bancos, de los cuales ninguno trabaja con un programa o taller para la primera infancia, y por otro lado, existen 64 Cooperativas de las cuales tan solo 4 de ellas tienen programas para los niños y niñas de 0 a 6 años: CREDIFACIL (Fundación Huellas), Ahorro y Crédito del Futuro, Ultrahuilca y Coonfie, estas dos últimas con las cuales se realizó la investigación.

Los talleres que manejan estas dos cooperativas para incluir a los niños son: fútbol, natación, pintura, danzas folklórica y moderna. Cada programa se realiza los fines de semana en las mañanas y los jueves en la jornada de la tarde, cuentan con una intensidad horaria de dos horas por semana y un número de participantes variable, entre 3 a 12 niños por taller.

Aunque estas dos cooperativas ofrecen programas para la primera infancia, se anota que el $28 \%$ de los padres de familia no se encuentran bien informados sobre que es la primera infancia y los programas que existen en las cooperativas, por lo que desconocen su importancia y beneficios para sus hijos.

Cada taller que se lleva a cabo en los programas cuenta con un profesor especializado en el área, en total son 7 los profesores de estas dos cooperativas. Para mencionar, los que trabajan la parte deportiva de los niños y niñas, realizan inicialmente un diagnóstico y preguntas de interés para elegir el deporte o deportes en los que van a practicar. El deporte favorece el buen desarrollo físico y mental de los niños, les ayuda a integrarse y adquirir valores fundamentales tales como la responsabilidad, potencia la creación y regularización de hábitos y además contribuye en el correcto desarrollo de sus huesos y músculos, su motricidad.

La labor que desempeñan los profesionales de estas cooperativas, aporta a la fluidez verbal, construcción de conocimiento, relaciones personales e interpersonales de los menores, dicho de otra manera y según lo expresado por María Montessori, la mente de los niños posee una capacidad maravillosa y 
única: la capacidad de adquirir conocimientos inconscientemente, pasando poco a poco del inconsciente a la conciencia, avanzando por un sendero en que todo es alegría. Se les compara con una esponja, con la diferencia que la esponja tiene una capacidad de absorción limitada, la mente del niño es infinita.

\section{Empresas Privadas: Pequeñín, Comfamiliar, Almacenes Éxito y Cencosud.}

Las empresas privadas que implementan programas para la primera infancia los enmarcan en dos tipos: unos asistenciales y otros pedagógicos, en los que integralmente se busca que los niños y niñas aprendan conocimientos y los pongan en práctica.

Dicha construcción de conocimiento transforma la realidad del infante, su entorno y cotidianidad, permitiéndole relacionarse con el ser, el saber y el saber hacer. Parafraseando a Vygotsky, psicólogo ruso, los programas dirigidos a la educación y formación del niño deben tener en cuenta el contexto en el que se desarrolla, pues este influye considerablemente y no debe ser ajeno a la constitución continua de identidad y cultura que hace el niño o niña.

En consideración, el conocimiento que los programas de empresas privadas aportan en beneficio de la primera infancia, cumplen una función social que en este caso se enfoca en la responsabilidad social.

Así, existe una empresa privada en la ciudad de Neiva conocida como Comfamiliar del Huila que ofrece dos programas de carácter pedagógico que contribuyen a la formación integral de la primera infancia y otra conocida como Pequeñín que benefician a una gran población infantil desde su periodo de gestación hasta su nacimiento. Mientras que otras empresas privadas como Almacenes Éxito y Cencosud contribuyen a la primera infancia con programas de carácter asistencial.

Los programas pedagógicos que ofrece Comfamiliar del Huila son CDI y el Colegio Los Lagos. El CDI tiene como fin es brindar un espacio adecuado, alegre y seguro con la orientación de profesionales de la psicología, la pedagogía y la nutrición al infante. Existen dos CDI en la ciudad de Neiva ubicados en la comuna 6 y comuna 8 en los barrios Bosques de San Luis y Panorama respectivamente. Son zonas en las que se evidencia vulnerabilidad en la población por el contexto social en la que se encuentran inmersos los niños. Por otro lado, el Colegio Los Lagos es un establecimiento dedicado a la formación y enseñanza de los niños de 3 a 5 años de edad, cuenta con profesionales de calidad que organizan jornadas de trabajo en las que incluyen estrategias que involucran la música, la danza y los juegos.

Por su parte, la empresa Pequeñín ofrece un programa llamado club pequeñín que se preocupa por ofrecer capacitaciones a madres gestantes sobre la estimulación y el cuidado del bebé a través de charlas que consisten en una actividad grupal ofrecida dentro o fuera de un establecimiento sobre un tema de interés de los participantes.

Las empresas que realizan programas asistenciales, como se nombró anteriormente, son Almacenes Éxito que tiene como programa una fundación que contribuye a la atención integral de más de 35000 niños, con aportes económicos para suplir las necesidades básicas en algunos hogares infantiles de la cuidad. El segundo programa de carácter asistencial es Cencosud que apadrina a una escuela y le brinda aportes económicos para alimentación, utensilios de aseo yútiles escolares.

Centros de estimulación temprana y recreación: Castillo mágico, Iwannabe y Baby'art.

La estimulación temprana es el conjunto de medios, técnicas y actividades con base científica y aplicada en forma sistemática y secuencial. Se emplea en niños desde su nacimiento hasta los 6 años con el objetivo de desarrollar al máximo sus capacidades cognitivas, físicas, emocionales y sociales, fortaleciendo el desarrollo integral. Se presenta entonces la necesidad de iniciar la estimulación de los niños desde el mismo momento de la concepción, entreviendo que "el hombre es un ser social y alcanza su desarrollo en las relaciones sociales que establece en ese medio a través de la actividad que desarrolla en él y la comunicación con los demás".

En Neiva, los centros de estimulación temprana son efectuados por el Castillo Mágico, Iwannabe, Baby Art's, quienes con programas tales como Estimulación temprana, Taller de adaptación al jardín, Taller de arte y diversión, Acompañamiento de tareas, Taller de danza de 4 a 6 años: Ballet, danza folclórica y moderna, servicio de cuidado nocturno y por horas; orientan el fortalecimiento de cada una de las dimensiones de desarrollo del niño, teniendo en cuenta los planteamientos de: Jean Piaget, que van al desarrollo sensoriomotor, cognitivo, social, entre otros.

La pertinencia de estos seis programas radica en su función alrededor de la necesidad del niño, con propósito de mejorar su desarrollo integral: motor, cognitivo, artístico, comunicativo y sicosocial; y fortalecer sus capacidades para lograr que sea independiente y creativo.

\section{Fundaciones: Fundación Corazón Valiente, Fundación Hermato Oncología del Huila, y Fundación Uniendo Sueños.}

Datos obtenidos del PNUD nos muestran que los niños en Colombia 16.722 .708 son el $41.5 \%$ de la población total, 6.500.000 viven en situación de pobreza $(38,9 \%)$,

4 - GRENIER, María Elena. La estimulación temprana: un reto del siglo XXI. Disponible en: http://www.campus-oei.org/celep/grenier.htm 
1.137.000 viven en situación de miseria (17,5\%). Las anteriores, cifras desalentadoras que deben propiciar el reconocimiento y cuidado de los niños para que los programas en primera infancia sean cada vez mayores, y cada actor social empiece a reconocer su propia responsabilidad y la necesidad de destinar esfuerzos y recursos para favorecer el desarrollo de los menores de 6 años tanto a mediano como a largo plazo.

Pese al panorama anterior, se integran acciones de fundaciones que convocan, desde sus propios roles y responsabilidades, el ejercicio del cumplimiento de los derechos de la primera infancia. Las fundaciones identificadas y que formaron parte de la población de esta investigación son Fundación Corazón Valiente, Fundación Hermato Oncología del Huila y Fundación Uniendo Sueños.

La Fundación Corazón Valiente tiene como objeto que el niño cuente con oportunidades que favorezcan su calidad de vida ofreciendo talleres de manualidades, mesas comunitarias y evangelización. Los talleres son dictados por personas de la comunidad, es decir, no tienen formación profesional enfocada a la primera infancia.

La Fundación Hermato Oncología del Huila, ofrece programas educativos en salud $y$ tratamientos médicos odontológicos para la primera infancia, cuenta con personal capacitado y especializado para atender esta población.

La Fundación Uniendo Sueños trabaja el programa ser nueve con madres gestantes y niños hasta los dos años de edad, haciendo énfasis en el apoyo nutricional y educación inicial, y enfatizando en la estimulación temprana con el programa góticas de la fundación éxito, y cuenta con personal capacitado en trabajo con primera infancia.

\section{ICBF: Hogares infantiles de Santa Inés, Guacirco, y Guambitario.}

La situación compleja que viven algunos niños y niñas y su vulnerabilidad los ha convertido en las principales víctimas de los conflictos armados, la pobreza, el maltrato físico y psicológico, entre otros; dificultades y problemáticas que develan la atención prioritaria que se debe tener hacía la primera infancia.

Siendo conscientes de que dichas situaciones cambian a largo plazo y con el involucramiento de toda la sociedad, lo que se traduce a un fuerte proceso de intervención, prevención, atención y sensibilización; en el marco de la protección a la niñez, el ICBF ha implementado programas que reducen los hechos que vulneran los derechos de la infancia. Uno de estos programas son los hogares infantiles, de los cuales se tomó una muestra de tres: Santa Inés, Guacirco y Guambitario.

Los tres hogares infantiles se caracterizan por estar ubicados en barrios populares de la ciudad, en donde predominan las familias de los estratos 1 y 2 . De esta forma la población que reciben es de bajos recursos económicos y en muchos casos con precarias condiciones de vida, por lo cual los bienestares se convierten en un espacio de protección y garantía de vida para muchos niños y niñas, por lo que se le resalta su carácter asistencialista.

En este sentido, se puntualiza con los actores de estos hogares que lo que se quiere alcanzar con estos programas es garantizar el desarrollo integral de los niños y niñas para brindarles espacios adecuados para su formación, pues se considera que los niños son una prioridad para llegar a mejorar la calidad de vida de las familias y en consecuencia la calidad de vida de la sociedad en general.

En la investigación se encontró que los tres hogares infantiles trabajan la estrategia de Cero a Siempre, Atención a la primera infancia (Salud, Nutrición, Valores, Proyectos pedagógicos, Lúdica y recreación, Calendario ambiental) y la fiesta de la lectura del ICBF.

Los docentes y directivos de los hogares tienen una misma visión encaminada al desarrollo integral de los niños y niñas favoreciéndolos en una buena y sana formación, además de garantizar todos sus derechos. Esta perspectiva docente muestra una buena aptitud profesional, pues además de la formación individual, las docentes tienen un claro enfoque de lo social y lo civil, encaminados a la construcción de la paz y la justicia social, que es el marco en el cual se desarrolla actualmente la Educación Inicial.

\section{Conclusiones}

Los programas ofrecidos por cada una de las entidades públicas y privadas se consideran de gran incidencia en el desarrollo y aprendizaje de los menores de seis años de edad, sin embargo existen barreas en su actuación que no permiten su continuidad e impacto, limitando a su vez la sistematización y evaluación de los mismos.

La trascendencia en el respeto y cumplimiento de los derechos de la primera infancia es una acción innegable de la sociedad, por lo tanto, el asunto es de interés púbico y el compromiso de carácter social.

"Los hechos de la responsabilidad social empresarial con la primera infancia se traducen en políticas y acciones concretas que favorezcan, apoyen y orienten, al interior de las organizaciones empresariales, a los padres y a las madres para que puedan cumplir en la vida cotidiana las responsabilidades con sus hijos y, hacia fuera de la empresa, apoyar y promover programas e iniciativas que igualen las oportunidades, desarrollen las potencialidades y capacidades de los menores de seis años, de manera tal que podamos decir que en Colombia, con el concurso de los empresarios, se fortalece la crianza amorosa, se produce conocimiento sobre la 
situación y necesidades de nuestros niños y niñas, se promueven iniciativas basadas en el juego, el arte, la literatura, se favorece la participación y la inclusión social $y$, en general, se garantiza un comienzo seguro a la vida para la construcción de una sociedad basada en valores democráticos, regida por principios de equidad y justicia social, pero sobre todo con mejores seres humanos". (Castañeda, 2011, p.6).

Los programas para la primera infancia expuestos, en su gran mayoría son entonces sin ánimo de lucro, no obstante se reconoce que el beneficio lucrativo de otros afecta la inclusión de los menores de estratos bajos, quienes no cuentan con un recurso económico para poder asistir a estos. A razón, su legitimación y exigibilidad es indispensable y deben estar caracterizados por el nivel de beneficios que le ofrecen al niño y las potencialidades físicas, mentales, sociales y afectivas que hacen desarrollen los niños y niñas.

Como conclusión final cabe resaltar que la mayoría de programas desarrollados cuentan con personal formado y capacitado, quienes se sienten complacidos y satisfechos llevando a cabo esta labor con los pequeños, pues se ven los resultados positivos conseguidos al enseñar sus conocimientos y al perseverar en la búsqueda de mejores resultados en el lugar donde laboran diariamente.

\section{Referentes bibliográficos}

Montero, R. (1990). "El contexto histórico de la obre de Vygostky: un enfoque socio-histórico", en Moll, Luis (Compilador), Vygostky y la Educación. Connotaciones y ?explicaciones de la psicología sociohistórica en la educación. Argentina: AIQUE

Código de la infancia y adolescencia. Art. 29 Ley 1098 del 2006

Castañeda, E. (2011). La importancia de la primera infancia. Instituto para el desarrollo y la innovación educativa. Recuperado de http://www.oei.es/idie/castaneda.pdf

Convención sobre los Derechos del Niño/Adoptada y abierta a la firma y ratificación por la Asamblea General en su resolución 44/25, de 20 de noviembre de 1989

Grenier, M. E. La estimulación temprana: un reto del siglo XXI. Disponible en: http://www.campus-oei.org/celep/grenier.htm

Gobierno Nacional. Estrategia de Cero a Siempre. Atención Integral a la Primera Infancia

Gobierno Nacional. Plan de Desarrollo Nacional 2010-2014. Prosperidad para todos. Disponible en: https://www.dnp.gov.co /PND/PND20102014.aspx

Margolis, F. (2013). Las experiencias de lenguaje en la primera infancia implican un adulto que canta, abraza, lee y descifra al otro." Entrevista con Yolanda Reyes. Mayo de 2013. Recuperado de http://www.imaginaria.com.ar/2013/05/yolanda-reyes/

Plan de Atención Integral a la Primera Infancia "Familia PiHuila, ¡Todos Corazón por la Primera Infancia!", 